\title{
Article \\ Route to Extend the Lifetime of a Discrete Time Crystal in a Finite Spin Chain without Disorder
}

\author{
Sayan Choudhury (D) \\ Department of Physics and Astronomy, University of Pittsburgh, Pittsburgh, PA 15260, USA; \\ sayan.choudhury@pitt.edu
}

check for

updates

Citation: Choudhury, S. Route to Extend the Lifetime of a Discrete Time Crystal in a Finite Spin Chain without Disorder. Atoms 2021, 9, 25. https://doi.org/10.3390/atoms9020025

Academic Editors: Masatoshi Kajita and James F. Babb

Received: 7 February 2021

Accepted: 10 April 2021

Published: 12 April 2021

Publisher's Note: MDPI stays neutral with regard to jurisdictional claims in published maps and institutional affiliations.

Copyright: (C) 2021 by the author. Licensee MDPI, Basel, Switzerland. This article is an open access article distributed under the terms and conditions of the Creative Commons Attribution (CC BY) license (https:/ / creativecommons.org/licenses/by/ $4.0 /)$.
Abstract: Periodically driven (Floquet) systems are described by time-dependent Hamiltonians that possess discrete time translation symmetry. The spontaneous breaking of this symmetry leads to the emergence of a novel non-equilibrium phase of matter-the Discrete Time Crystal (DTC). In this paper, we propose a scheme to extend the lifetime of a DTC in a paradigmatic model-a translation-invariant Ising spin chain with nearest-neighbor interaction $J$, subjected to a periodic kick by a transverse magnetic field with frequency $\frac{2 \pi}{T}$. This system exhibits the hallmark signature of a DTC - persistent sub-harmonic oscillations with frequency $\frac{\pi}{T}$-for a wide parameter regime. Employing both analytical arguments as well as exact diagonalization calculations, we demonstrate that the lifetime of the DTC is maximized, when the interaction strength is tuned to an optimal value, $J T=\pi$. Our proposal essentially relies on an interaction-induced quantum interference mechanism that suppresses the creation of excitations, and thereby enhances the DTC lifetime. Intriguingly, we find that the period doubling oscillations can last eternally in even size systems. This anomalously long lifetime can be attributed to a time reflection symmetry that emerges at $J T=\pi$. Our work provides a promising avenue for realizing a robust DTC in various quantum emulator platforms.

Keywords: time crystals; Floquet systems; time translation symmetry breaking; quantum many-body echo

\section{Introduction}

The classification of phases of matter based on symmetries and symmetry breaking forms one of the cornerstones of modern statistical physics [1]. Crystals represent a ubiquitous example of this paradigm, where inter-atomic interactions lead to the breaking of spatial translation symmetry [2]. In a seminal paper in 2012, Frank Wilczek extended the concept of symmetry breaking to the time domain [3]. In particular, he postulated that the ground state of attractively interacting bosons on a Aharonov-Bohm ring can exhibit periodic oscillations in time; this novel phase of matter was dubbed a "Quantum Time Crystal", since it breaks time translation symmetry. Wilczek's bold proposal generated considerable excitement and debate [4-7]. Eventually however, the existence of quantum time crystals in equilibrium was ruled out by a no-go theorem [8]. This crucial insight has prompted several researchers to explore the possibility of realizing time crystals in non-equilibrium quantum systems [9-26]. These efforts have been immensely fruitful and led to the development of an active area of research [27-39].

A particularly profound development in this field has been the discovery of discrete time crystals in periodically driven (Floquet) quantum many-body systems [10-13]. A Floquet system is described by a time-periodic Hamiltonian, $H(t)$ where $H(t+T)=H(t)$. A discrete time crystal (DTC) is an out-of-equilibrium phase of matter that breaks this time translation symmetry, and consequently shows a stable sub-harmonic response of physical observables. In particular, the DTC phase is characterized by the existence of a class of observables $O$ and initial states, $|\psi\rangle$, such that $\langle\psi|O(t)| \psi\rangle \neq\langle\psi|O(t+T)| \psi\rangle$. Furthermore, to qualify as a genuine non-equilibrium phase of matter, these sub-harmonic oscillations must persist at long times $(t / T \gg 1)$, without fine tuning the Hamiltonian 
parameters $[40,41]$. Through several theoretical and experimental studies, the existence of the DTC phase has now been firmly established [40-56].

The first realization of a DTC crucially relied on the presence of many-body localization (MBL) [42]. This is because Floquet many-body localized systems do not absorb energy from the drive, and consequently evades thermalization. This leads to persistent oscillations of physical observables with a characteristic frequency, $\omega \neq \frac{2 \pi}{T}$ at long times. However, the requirement of MBL is very restrictive, thereby making the realization of a DTC in large systems extremely challenging $[57,58]$. Furthermore, MBL can lead to long-lived transient dynamics, thereby making it difficult to access the long-time behavior of the system in current experiments [59]. These issues have prompted researchers to examine other routes for realizing a robust DTC.

Intriguingly, recent research efforts have shown that it is possible to realize a robust DTC in the absence of disorder [40,41,52-56]. This surprising observation implies the presence of mechanisms other than MBL that can prevent Floquet systems from heating up. Such mechanisms may also be useful for stabilizing other non-equilibrium phases of matter. To explore this issue in a concrete example, we analyze the conditions necessary for creating a time crystal in a periodically driven Ising spin chain. Yu et al. have already demonstrated that this system can exhibit discrete time-translation-symmetry-breaking (TTSB) and argued that the DTC order is stabilized by high-frequency driving [60]. High-frequency driving can also be used to realize prethermal time crystals [14-16,59]. Unfortunately, when the driving frequency is very high, the DTC order can be destroyed due to dissipative coupling to higher bands [61]. This necessitates the search for other pathways to stabilize time crystals.

It has been recently demonstrated that Floquet phases of matter can be stabilized by a many-body quantum interference, thereby circumventing the problems associated with high-frequency driving [62-64]. In particular, Lyu et al. have shown that it is possible to realize an eternal DTC in a periodically driven, even size, infinite range interacting Ising spin chain, by appropriately tuning the interaction strength [64]. This naturally raises the question of whether a similar scheme can be used to stabilize a DTC in a kicked short-range Ising model. We answer this question affirmatively, and derive the optimal interaction strength that maximizes the DTC lifetime in a finite chain. An important aspect of our scheme is that the optimal interaction parameter maximizes the lifetime for both even and odd size chains, while the protocol presented in ref. [64] is only applicable for even size chains. It is interesting to note that for this optimal interaction strength $J$, strong disorder leads to thermalization. Our results provide a novel route for extending the lifetime of DTCs in translation-invariant systems, and can guide the experimental realizations of DTCs [65-68].

Before proceeding further, we note that the phenomenology of DTCs is a closely related to the dynamical Casimir effect, where periodic modulation of boundaries or material properties of a system leads to a parametric amplification of initial vacuum fluctuations $[69,70]$. A particularly striking example of this phenomenon is the temporal Bragg diffraction, where a periodic modulation of the effective charge of neutral atoms leads to phonon backscattering in time. In this case, the modulation frequency $\omega$ is related to the initial phonon frequency $\omega_{c}$ as: $\omega=(n \pm 1) \omega_{c} / 2$, where $n$ is an integer [71,72]. This is analogous to the discrete TTSB that characterizes DTCs.

This paper is organized as follows. In Section 2, we introduce our model and discuss the conditions under which the system behaves like a discrete time crystal. In Section 3, we describe our scheme to extend the DTC lifetime by tuning the Ising interaction. In particular, we analytically derive the optimal interaction strength that maximizes the lifetime of the DTC. Furthermore, we establish the validity of our analytical results by numerically analyzing the dependence of the lifetime on the interaction strength as well as the system size. Thus, our calculations provide a general framework to realize time crystals in disorder free spin chains. Finally, we discuss potential realizations of our model. We conclude with a brief summary of our results in Section 4 . 


\section{Model}

We study the time evolution of a 1D spin chain described by the following Floquet Hamiltonian:

$$
H=\sum_{i}\left(J S_{i}^{z} S_{i+1}^{z},+(\pi-2 \epsilon) S_{i}^{x}, \delta(t-n T)\right),
$$

where $J$ is the nearest-neighbor Ising interaction, $\vec{S}^{i}=\frac{\hbar}{2} \vec{\sigma}^{i}$ and $\vec{\sigma}^{i}$ are the Pauli spin matrices (see Figure 1).
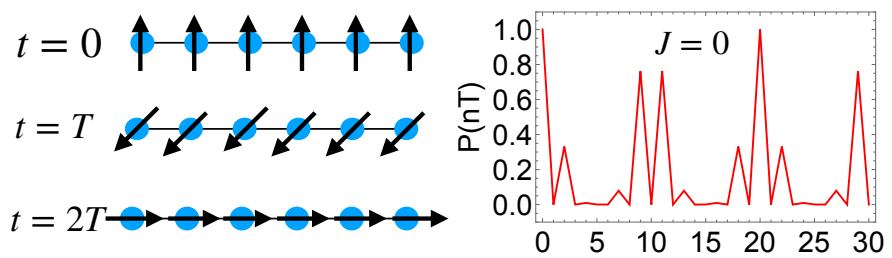

(a)

$\mathrm{n}$
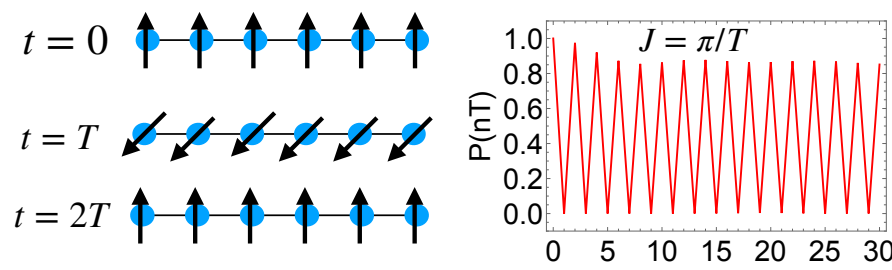

(b)

Figure 1. (a) The return probability, $P(n T)$ (defined in Equation (3)) when $J=0$. When the $\pi$ pulse is imperfect, then there is no oscillation of the order parameter and hence no time crystal. (b) The return probability, $P(n T)$ when $J T=\pi$ for the nearest-neighbor Ising model. This example clearly shows that persistent time crystal order can be established in a disorder free spin chain by tuning the interaction strength.

Setting $\hbar=1$, we find the time-evolution operator for one Floquet period to be:

$$
U(T)=e^{(-i H T)} \equiv e^{\left(-i \frac{I T}{4} \sum_{i} \sigma_{i}^{z} \sigma_{i+1}^{z}\right)} e^{\left(-i\left(\frac{\pi}{2}-\epsilon\right) \sum_{i} \sigma_{i}^{x}\right)} .
$$

When $\epsilon=0$, the spin chain trivially exhibits time correlations at twice the driving frequency for any initial state that spontaneously breaks the $\mathbb{Z}_{2}$ symmetry of $H_{0}$. However, to qualify as a DTC, there should be a class of initial states for which some physical observables must exhibit indefinitely long sub-harmonic oscillations in the thermodynamic limit, even when $\epsilon \neq 0[40,41]$. This kind of TTSB occurs in our model, when the Ising interaction is non-zero [60].

In this paper, we study the response of the spin chain to the periodic drive by computing the return probability:

$$
P(t)=|\langle\psi(t) \mid \psi(0)\rangle|^{2}
$$

If $P(t)$ exhibits robust sub-harmonic oscillations for long times, we conclude that the system is in the DTC phase. In the DTC phase, the stroboscopic return probability at times $2 n T$ remains almost constant for a very long time, and the DTC lifetime, $n^{*}$ is usually defined to be the number of drive periods, after which $P(2 n T)$ falls below a critical value $(\sim 0.05)[40,51,67]$. The rationale for using this measure to quantify lifetime is the following: the Fourier transform of $P(t)$ taken up to a time $t<2 n^{*} T$ exhibits a peak at $\omega / 2$, whereas this peak splits when the Fourier transform is taken over longer times; thus, the DTC exhibits persistent sub-harmonic oscillations at a rigid rhythm up to a time, $t \sim 2 n^{*} T$ (see Figure 2). In the next section, we systematically explore the dependence of the DTC lifetime on the Ising interaction strength. 

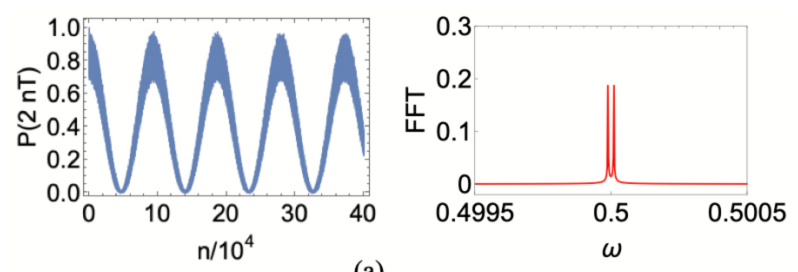

(a)
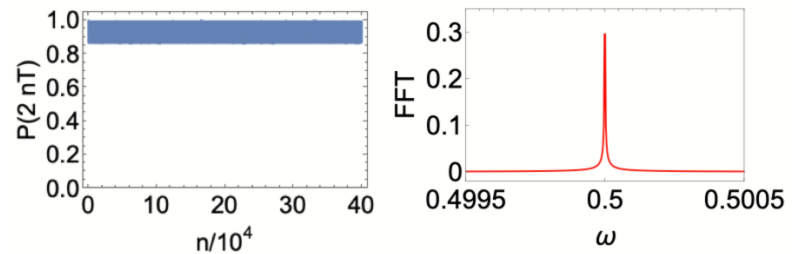

(b)

Figure 2. The Fourier transform of the stroboscopic return probability, $P(2 n T)$ for a 10-site chain with (a) $J T=\frac{\pi}{2}$ and (b) $J T=\pi$. As exemplified by the case of $J T=\frac{\pi}{2}, P(2 n T)$ oscillates when $n>n^{*}$ ( $n^{*}$ is the DTC lifetime defined below Equation (3)). These oscillations result in a splitting of the $\omega / 2$ Fourier peak.

\section{Maximizing the DTC Lifetime}

In this section, we study the stroboscopic evolution of a $L$-site periodic chain, prepared in a spin-polarized initial state i.e., $|\psi(t=0)\rangle$ can be either $|\ldots \uparrow \uparrow \uparrow \ldots\rangle$ or $|\ldots \downarrow \downarrow \downarrow \ldots\rangle$. We analyze the response of the system to imperfect $\pi$-pulses in the experimentally relevant parameter regime, where $\sin (\epsilon) \approx \epsilon$ and $\cos (\epsilon) \approx 1-\frac{\epsilon^{2}}{2}$.

\subsection{Few-Cycles Dynamics}

To the lowest order in $\epsilon$, we obtain the following expression for the return probability after the first two pulses:

$$
\begin{aligned}
P(2 T) & =\left|\cos (\epsilon)^{(2 L)}-L \exp (-i J T) \cos (\epsilon)^{(2 L-2)} \sin (\epsilon)^{2}\right|^{2} \\
& \approx\left|\left(1-\frac{\epsilon^{2}}{2}\right)^{(2 L)}-L \exp (-i J T)\left(1-\frac{\epsilon^{2}}{2}\right)^{(2 L-2)}(\epsilon)^{2}\right|^{2} \\
& \approx\left|1-L \epsilon^{2}(1+\exp (-i J T))\right|^{2} .
\end{aligned}
$$

It is straightforward to see that in this limit, an almost perfect revival of the initial state occurs when $J T=\pi$. This revival implies that the system can exhibit persistent oscillations of physical order parameters (like the magnetization) with a frequency $\frac{\pi}{T}-\mathrm{a}$ direct signature of discrete TTSB.

This remarkable conclusion can be understood by a very simple physical picture. Let us assume that the system is initially prepared in a spin-polarized state: $|\psi(t=0)\rangle=$ $|\ldots \uparrow \uparrow \uparrow \ldots\rangle$. Thus, after one imperfect $\pi$ pulse, the state of the system is:

$$
\begin{aligned}
\left|\psi\left(t=T^{+}\right)\right\rangle \approx & \cos (\epsilon)^{L}|\ldots \downarrow \downarrow \downarrow \ldots\rangle-\cos (\epsilon)^{L-1} \sin (\epsilon) \\
& \sum_{h}|\ldots \downarrow \ldots \downarrow \uparrow \downarrow \downarrow \ldots \downarrow \ldots\rangle
\end{aligned}
$$

Just before the second $\pi$ pulse is applied, the spin chain wavefunction is :

$$
\begin{array}{ccc}
\left|\psi\left(t=2 T^{-}\right)\right\rangle & \approx \quad \cos (\epsilon)^{L}|\ldots \downarrow \downarrow \downarrow \ldots\rangle-\cos (\epsilon)^{L-1} \sin (\epsilon) \\
& e^{-i J T} \quad \sum_{h}|\ldots \downarrow \ldots \downarrow \uparrow \downarrow \downarrow \ldots \downarrow \ldots\rangle
\end{array}
$$


Now, after the second $\pi$ pulse is applied the state of the system becomes:

$$
\begin{aligned}
\left|\psi\left(t=2 T^{+}\right)\right\rangle= & \left(\cos (\epsilon)^{(2 L)}-L e^{(-i J T)} \cos (\epsilon)^{(2 L-2)} \sin (\epsilon)^{2}\right) \\
& |\psi(t=0)\rangle+\ldots
\end{aligned}
$$

Thus, when the condition $J T=\pi$ is satisfied, $\left|\psi\left(t=2 T^{+}\right)\right\rangle \approx|\psi(t=0)\rangle$. In other words, a revival of the initial state occurs after two pulses due to a constructive quantum interference. A schematic derivation of this result is illustrated in Figure 3. Thus, an interaction-induced quantum interference can enable the creation of a DTC - a phenomena similar to the recently proposed many-body echo [62,63]. A similar scheme has been studied for the infinite range interacting Ising model [64]; in that case $J T=\pi$ leads to a perfect revival of the initial state for even size chains, while there is no DTC order for odd size chains. However, for the nearest-neighbor Ising model, $J T=\pi$ is the optimal interaction strength for both odd and even size chains.

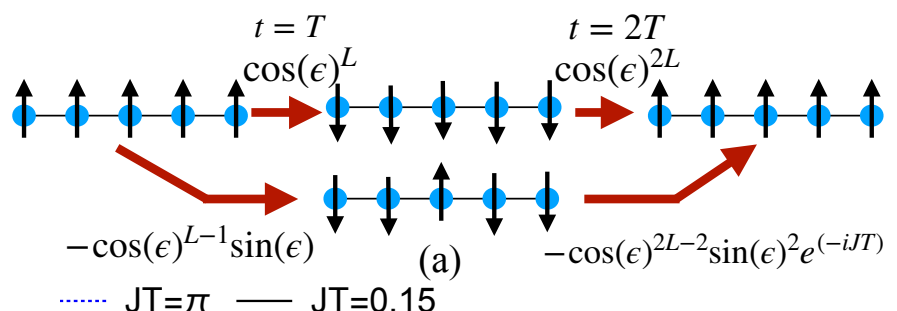

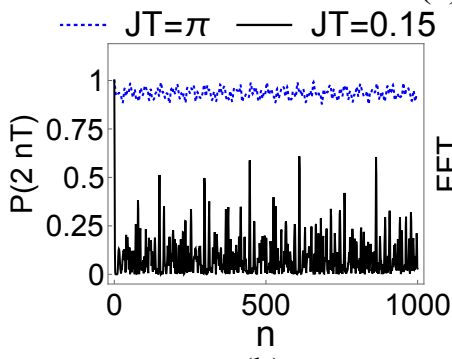

(b)

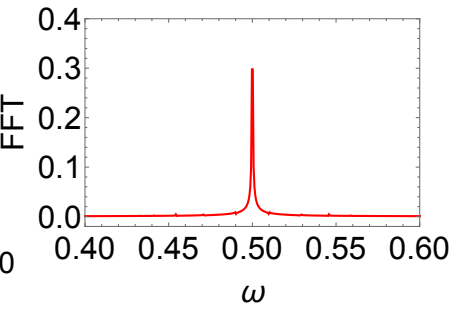

(c)

Figure 3. (a) Illustration of the state of the spin chain after two imperfect $\pi$ pulses, starting from a fully spin-polarized state. After the first pulse the spin chain wavefunction primarily comprises two classes of states: A fully spin-polarized state, and $L$ one-magnon states (i.e., a state with $L-1$ spin-downs and one spin-up). When $J T=\pi$, the two pathways interfere constructively, leading to a revival of the initial state. (b) The stroboscopic return probability $P(2 n T)$ for two different values of $J T$, when $\epsilon=0.07 \pi$. Persistent oscillations can be observed when $J T=\pi$. (c) The Fourier spectrum of the return probability shows a robust peak at $\omega_{0} / 2$, where $\omega_{0}=\frac{2 \pi}{T}$, when $J T=\pi$.

\subsection{Long-Time Dynamics}

A perfect revival of the initial state would lead to an eternal time crystal. However, in our model, the revival is imperfect, and the discrete time crystalline order may disappear at later times. To understand what happens to the DTC at later times, we first study the dynamics of the spin chain in the few-cycles regime. In this case, when $\epsilon$ is small, the evolution of the spin chain can be captured by an effective analytic model [60]. This effective model is obtained by truncating the Hilbert Space to only two kinds of states: (a) Fully polarized states, and (b) One-magnon states i.e., states with $(L-1)$ spin-ups (spin-downs), and 1 spin-down (spin-up). Using this effective model, we find that the spin chain wavefunction after $2 n$ pulses is given by:

$$
|\psi(2 n T)\rangle=c_{0}(2 n T)\left|\psi_{0}\right\rangle+c_{1}(2 n T) \sum_{h}|h\rangle,
$$


where $\left|\psi_{0}\right\rangle=|\ldots \uparrow \uparrow \uparrow \ldots\rangle$ (the fully polarized state), $|h\rangle=\left|\ldots \uparrow \ldots \uparrow \downarrow_{h} \uparrow \ldots \uparrow \ldots\right\rangle$ (the one-magnon states), and

$$
c_{1}(2 n T)=\epsilon \sum_{j=1}^{2 n T-1} \exp (-i j J T) .
$$

This implies that

$$
\left|c_{1}(2 n T)\right|=\epsilon\left|\frac{\sin (n J T)}{\sin (T J /(2))}\right|,
$$

and thus, the stroboscopic return probability $P(2 n T)$ is given by:

$$
P(2 n T)=c_{0}(2 n T)^{2}=1-L \epsilon^{2}\left(\frac{\sin (n J T)}{\sin (T J /(2))}\right)^{2} .
$$

When $J T=0$, the return probability decays very fast, implying the absence of any TTSB. In the presence of interactions however, the spins can become synchronized, and the time crystal order can persist. Furthermore, when $J T=\pi, P(2 n T) \approx 1$, thereby indicating that the system may exhibit time crystalline behavior for long times.

Next, we investigate the fate of the system at even longer times, by performing exact diagonalization calculations on a 11-site model, when $\epsilon=0.1 \pi$. Our results are shown in Figure 4. It is evident from this figure that while there is a large parameter regime, where the spin chain exhibits sub-harmonic oscillations at a frequency of $\pi / T$, the DTC lifetime is maximized when $J T=\pi$. To further substantiate our finding, we analyze the dependence of the DTC lifetime, $n^{*}$ on system size for some fixed values of $J T$. As shown in Figure $5 \mathrm{a}$, we find that $n^{*}$ increases exponentially with the system size. This is a characteristic feature of DTCs. Interestingly, we find that when $J T \approx \pi$, the lifetime of even size chains of length $L$ can be greater that of odd size chains of length $L+1$. As we shall explain below, this behavior can be understood as a consequence of a time reflection symmetry of the model at $J T=\pi$. It is also worth noting that the behavior of $P(2 n T)$ is symmetric around $J T=\pi$, thereby implying that $n^{*}$ is the same for $J T=y$ and $J T=2 \pi-y$, where $y \in \mathbb{R}$. We provide numerical evidence for this result in Figure $5 b$. The results from Figure 5 suggests the existence of a critical $J$, below which TTSB would not occur for every value of $\epsilon$. We proceed to determine the region of parameter space, where the system is a DTC by evaluating the average stroboscopic return probability, $\overline{P(2 n T)}$ over 1000 periods. Our results are shown in Figure 6b.

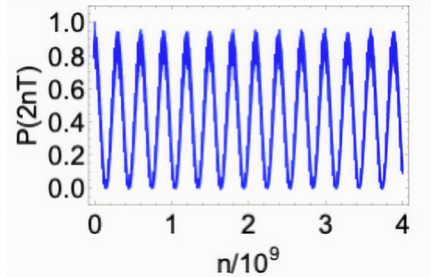

(a)

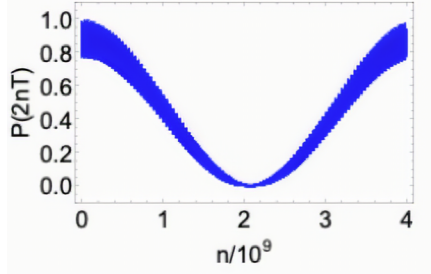

(d)

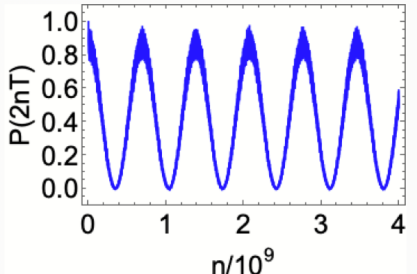

(b)

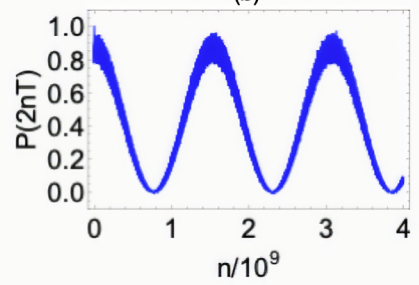

(e)

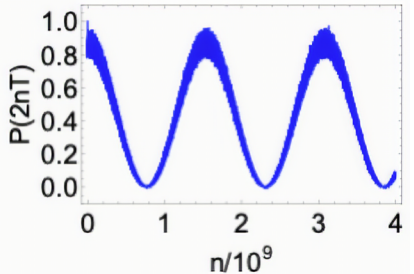

(c)

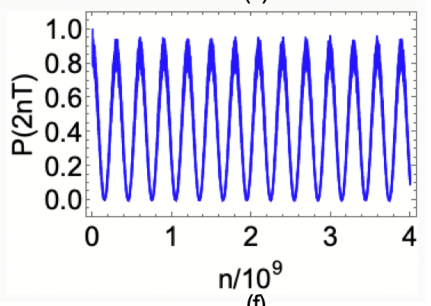

(f)

Figure 4. (a-f): The stroboscopic return probability at times $2 n T(P(2 n T))$ for a 11-site spin chain with nearest-neighbor interactions; $\frac{J T}{\pi}=0.75,0.85,0.95,1,1.05$, and 1.25 respectively, and $\epsilon=0.1 \pi$. We find that time crystal signatures can be observed for a wide range of $J T$, and the time crystal lifetime is maximum when $J T=\pi$. 


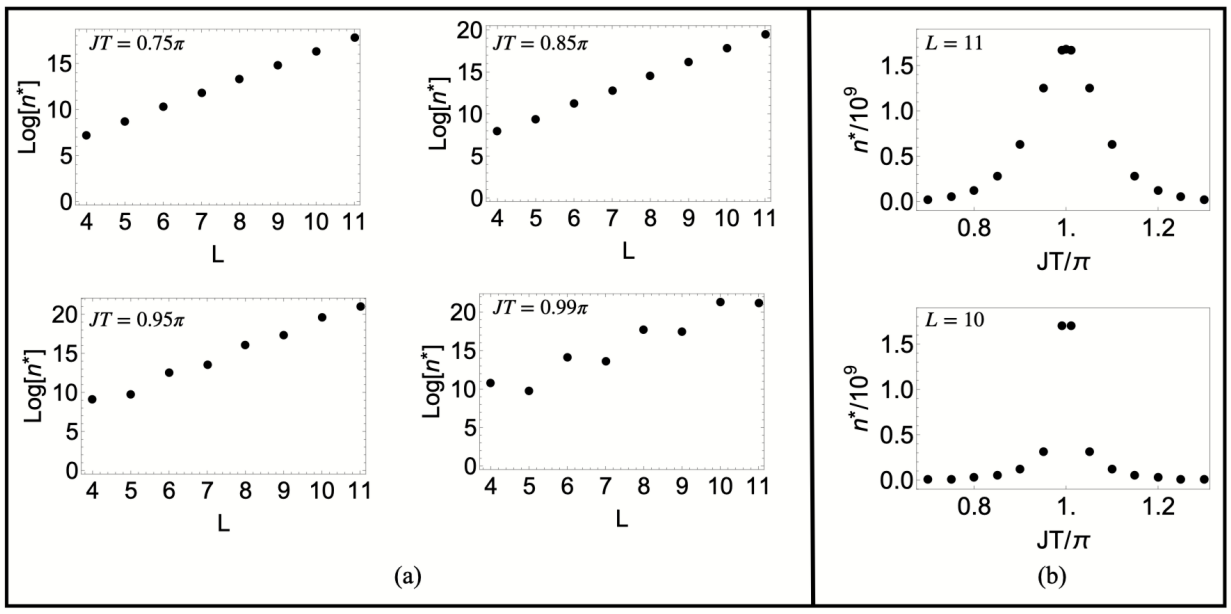

Figure 5. (a) The dependence of the DTC lifetime, $n^{*}$ defined below Equation (3) on the system size for various values of $J T$. We find that the lifetime increases exponentially with the system size. When $J T \approx \pi$, the spin chain exhibits an interesting feature: the lifetime of an even size system of length $L$ is almost the same as that of an odd size system of length $L+1$. This is due to a constructive interference from several paths for even size chains. (b) The dependence of the lifetime on JT for a fixed spin chain length, $L$. It is evident that $n^{*}$ is maximum, when $J T=\pi$ for both even and odd size chains.

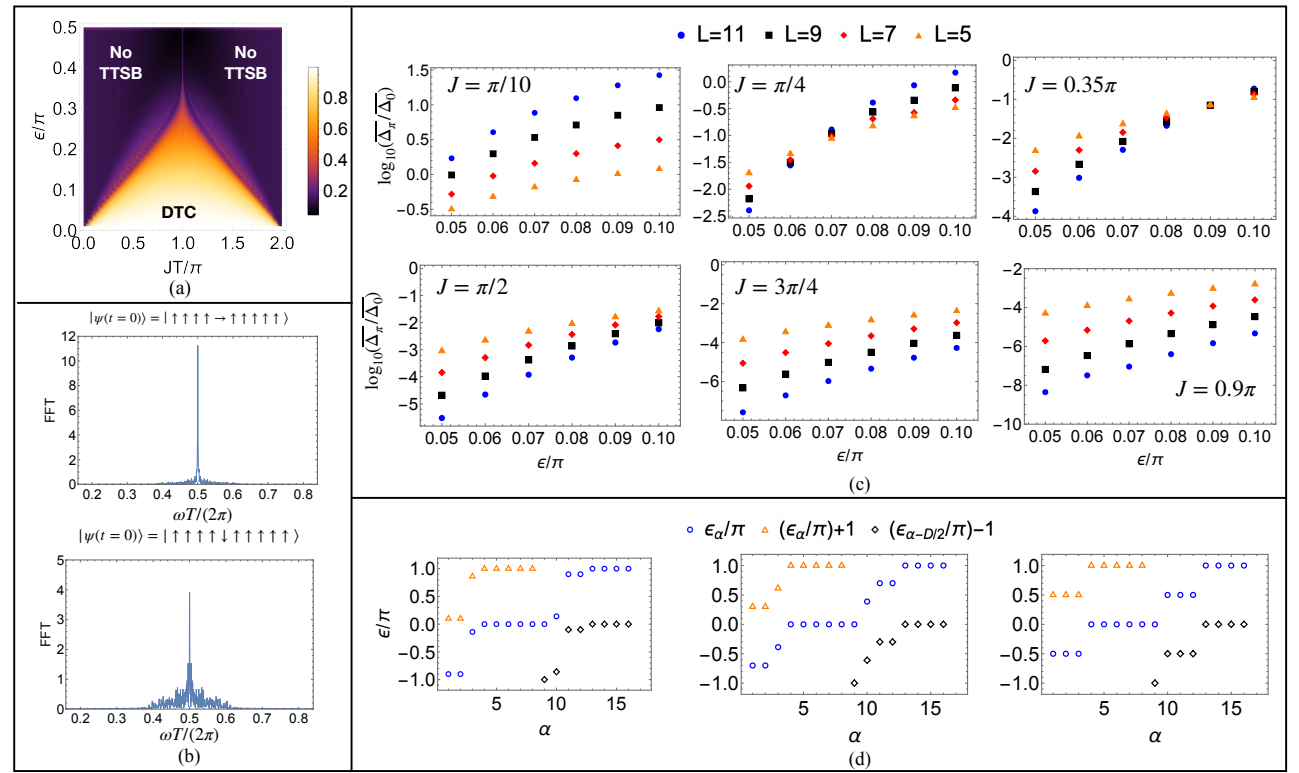

Figure 6. (a) Phase diagram of the model obtained from averaging $P(2 n T)$ over 1000 periods for a 10-site system. There is a large region of parameter space where the system exhibits DTC order. (b) The Fourier spectrum of $\sigma_{i}^{z}(t)$ when the $i$-th spin is polarized along the $+x$ and $-z$ direction respectively, while the other spins are polarized along $+z$ direction. It is clear that the system exhibits a sharp Fourier peak at $\omega=\pi / T$ - a direct signature of period doubling oscillations. (c) The eigenstate averaged energy gaps defined in Equations (12) and (13) as a function of $\epsilon$ and $J$ for different system sizes $L$. In the DTC regime, $\frac{\Delta_{\pi}}{\Delta_{0}} \rightarrow 0$ in the thermodynamic limit. It is evident from this figure that the transition from the no-TTSB phase to the DTC phase is accompanied by a transition from increasing $\frac{\Delta_{\pi}}{\Delta_{0}}$ to decreasing $\frac{\Delta_{\pi}}{\Delta_{0}}$ with increasing system size. (d) Quasi-energy spectrum for a 4-site system when $J T=\pi$ : there are 4 perfect $\pi$-spectrally paired states due to the time reflection symmetry that emerges at this point. These perfect $\pi$-spectral pairs would lead to eternal period doubling oscillations. 
Our results so far have focused on the period doubling oscillations exhibited by the spin chain, when it is initialized in a globally polarized state. Although these oscillations suggest that the system may be a DTC, to rigorously establish the existence of the DTC phase, we must examine whether the model possesses two crucial characteristics: (a) it must be integrable, and (b) it must host an extensively large number of eigenstate pairs with quasi-energies differing by $\pi / T$. The integrability of this system is already well established $[73,74]$; this feature allows it to evade thermalization. To check for the " $\pi$ spectral pairing", we compute the energy difference between neighboring eigenstates:

$$
\Delta_{0}=\epsilon_{i+1}-\epsilon_{i}
$$

as well as

$$
\Delta_{\pi}=\epsilon_{i+D / 2}-\epsilon_{i}-\pi / T,
$$

where $\epsilon$ is the quasi-energy and $D=2^{L}$ is the Hilbert Space dimensions. Robust " $\pi$-spectral pairing" occurs, when $\Delta_{\pi} \ll \Delta_{0}$ [11]. Our results are shown in Figure 6c; it is quite clear that the model hosts an extensive number of $\pi$-spectral paired eigenstates in the parameter regime that we had earlier identified to be a DTC. Consequently, we find that the system can exhibit period doubling oscillations for different initial states (see Figure 6b). This analysis of the quasi-energy spectrum clearly establishes this model as a DTC.

Finally, we address the "odd-even" effect in the DTC lifetime, when $J T=\pi$. Intriguingly at this special point, the system possesses a time reflection symmetry generated by the operator [75]:

$$
R=\prod_{i=1}^{L} \sigma_{i}^{x} \prod_{j=1}^{L} \sigma_{j}^{z}
$$

such that

$$
R U(T) R^{\dagger}=\exp (-i L \pi / 2) U(T),
$$

where $U(T)$ is the Floquet propagator defined in Equation (2).

As a consequence of this symmetry, there are at least $2^{L / 2}$ states exactly at quasienergies 0 and $\pi$ for even size systems (for a detailed derivation of this result, see ref. [75]). This result implies that there are at least $2^{L / 2}$ exact $\pi$-spectral paired eigenstates even in a finite size system (see Figure 6d). We note that generically such exact $\pi$-spectral pairing occurs only in thermodynamic limit. The presence of these exact $\pi$-spectral pairs has a striking consequence: the spin chain can exhibit eternal period doubling oscillations. To see this, let us assume that the system is initially prepared in a state:

$$
|\psi(t=0)\rangle=\left|\bar{\psi}_{ \pm}\right\rangle=\left|\phi_{0}\right\rangle \pm\left|\phi_{\pi}\right\rangle,
$$

where $\left.\phi_{0}\right\rangle\left(\phi_{\pi}\right\rangle$ is a Floquet eigenstate with quasi-energy $0(\pi)$. In this case:

$$
|\psi(t=T)\rangle=\left|\bar{\psi}_{\mp}\right\rangle=\left|\phi_{0}\right\rangle \mp\left|\phi_{\pi}\right\rangle
$$

and

$$
|\psi(t=2 T)\rangle=\left|\bar{\psi}_{ \pm}\right\rangle=\left|\phi_{0}\right\rangle \pm\left|\phi_{\pi}\right\rangle=|\psi(t=0)\rangle
$$

This perfect revival after two pulses implies that the stroboscopic return probability, $P(2 n T)$ is always 1 and the DTC has eternal lifetime. Of course, the states $\left|\bar{\psi}_{ \pm}\right\rangle$is generally difficult to prepare experimentally. However, these eternal oscillations can be observed as long as the initial state has a significant overlap with $\left|\bar{\psi}_{ \pm}\right\rangle$. For the finite size systems considered here, the globally polarized state is indeed an example of these special class of initial states, and therefore the chain behaves as an eternal DTC, when it is initialized in this state. Furthermore, the lifetime of the DTC in even size systems is significantly enhanced when $|J T-\pi| \ll 1$.

A natural question to investigate at this point is the effect of disorder on this system, when $J T \approx \pi$. By comparing to known results in the literature, we conclude that the 
presence of strong onsite disorder would destroy the DTC in this parameter regime [12]. A drawback of our scheme is that an eternal DTC can only be realized for a limited class of initial states. This drawback can be overcome by engineering infinite range interactions [64].

\title{
3.3. Experimental Realization
}

Before we conclude, it is instructive to discuss potential experimental realizations of our proposal. A promising platform to test our predictions is a Rydberg atom chain [67]. In this system, the atomic ground state and a chosen Rydberg state can be mapped to a pseudospin- $1 / 2$ system; the periodic kick can be simulated using a periodically modulated laser beam that couples these states [76-78]. Alternatively, this model can be engineered using ultracold bosons loaded in a tilted optical lattice in the Mott insulator regime [79]. Finally, we note that digital quantum simulations on superconducting quantum processors $[80,81]$ and trapped ion quantum computers [82,83] provide an alternative route to realize the long-lived DTC we have studied in this paper.

\section{Summary and Outlook}

A time crystal is an intriguing non-equilibrium phase of matter that is expected to be a very useful platform for performing precision measurements $[84,85]$ and quantum simulation [86]. In this paper, we have described a scheme to extend the lifetime of a DTC in a periodically driven finite Ising spin chain with nearest-neighbor interactions and no disorder. Our major insight is that the lifetime of the DTC can be maximized by tuning the interaction strength to an optimal value. This is a consequence of a novel interactioninduced quantum interference mechanism. Furthermore, we find that the DTC lifetime grows exponentially with the system size, with a considerably greater enhancement for even size chains at the optimal interaction strength. Finally, we have discussed possible realizations of our model in various quantum simulator platforms.

One of the grand challenges of Floquet engineering is to determine an optimal frequency window, where heating is suppressed [87]. Our scheme can overcome problems associated with high-frequency driving, and thus it can play an important role in designing future experiments on time crystals. Although we have considered the case of nearest-neighbor interactions in this paper, it will be interesting to extend our treatment to long-range interacting systems in the future. In an exciting development, Viebahn et al. have recently demonstrated that a similar two path interference can be employed to suppress heating in a periodically driven ultracold fermionic system [88]. This leads us to believe that generalizations of our scheme may be useful for stabilizing other Floquet systems, and we plan to explore this in future work.

Funding: This work is supported by the AFOSR Grant No. FA9550-16-1-0006, and the MURI-ARO Grant No. W911NF17-1-0323 through UC Santa Barbara.

Institutional Review Board Statement: Not applicable.

Informed Consent Statement: Not applicable.

Data Availability Statement: The data that support the findings of this study are available from the corresponding author upon reasonable request.

Acknowledgments: The author thanks Qi Zhou for discussions and encouragement, and the Wilczek Quantum Center for its hospitality while part of this work was performed.

Conflicts of Interest: The authors declare no conflict of interest.

\author{
Abbreviations \\ The following abbreviations are used in this manuscript: \\ DTC Discrete Time Crystal \\ TTSB Time Translation Symmetry Breaking \\ MBL Many-Body Localization
}




\section{References}

1. Landau, L.D.; Lifshitz, E.M. Course of Theoretical Physics; Pergamon: Oxford, UK, 1980; Volume 5.

2. Beekman, A.J.; Rademaker, L.; van Wezel, J. An introduction to spontaneous symmetry breaking. SciPost Phys. Lect. Notes 2019, 11, 21468. [CrossRef]

3. Wilczek, F. Quantum Time Crystals. Phys. Rev. Lett. 2012, 109, 160401. [CrossRef] [PubMed]

4. Li, T.; Gong, Z.X.; Yin, Z.Q.; Quan, H.; Yin, X.; Zhang, P.; Duan, L.M.; Zhang, X. Space-Time Crystals of Trapped Ions. Phys. Rev. Lett. 2012, 109, 163001. [CrossRef] [PubMed]

5. Bruno, P. Impossibility of Spontaneously Rotating Time Crystals: A no-go theorem. Phys. Rev. Lett. 2013, 111, 070402. [CrossRef] [PubMed]

6. Thomas, J. The Aftermath of a Controversial Idea. Physics 2013, 6, 31.

7. Nozières, P. Time Crystals: Can Diamagnetic Currents Drive a Charge Density Wave into Rotation? EPL 2013, 103, 57008. [CrossRef]

8. Watanabe, H.; Oshikawa, M. Absence of Quantum Time Crystals. Phys. Rev. Lett. 2015, 114, 251603. [CrossRef]

9. Sacha, K. Modeling Spontaneous Breaking of Time-Translation Symmetry. Phys. Rev. A 2015, 91, 033617. [CrossRef]

10. Khemani, V.; Lazarides, A.; Moessner, R.; Sondhi, S.L. Phase Structure of Driven Quantum Systems. Phys. Rev. Lett. 2016, 116, 250401. [CrossRef]

11. von Keyserlingk, C.W.; Khemani, V.; Sondhi, S.L. Absolute Stability and Spatiotemporal Long-Range Order in Floquet Systems. Phys. Rev. B 2016, 94, 085112. [CrossRef]

12. Yao, N.Y.; Potter, A.C.; Potirniche, I.D.; Vishwanath, A. Discrete Time Crystals: Rigidity, Criticality, and Realizations. Phys. Rev. Lett. 2017, 118, 030401. [CrossRef]

13. Else, D.V.; Bauer, B.; Nayak, C. Floquet Time Crystals. Phys. Rev. Lett. 2016, 117, 090402. [CrossRef]

14. Else, D.V.; Bauer, B.; Nayak, C. Prethermal Phases of Matter Protected by Time-Translation Symmetry. Phys. Rev. X 2017, 7, 011026. [CrossRef]

15. Machado, F.; Else, D.V.; Kahanamoku-Meyer, G.D.; Nayak, C.; Yao, N.Y. Long-range prethermal phases of nonequilibrium matter. Phys. Rev. X 2020, 10, 011043. [CrossRef]

16. Zeng, T.S.; Sheng, D. Prethermal time crystals in a one-dimensional periodically driven Floquet system. Phys. Rev. B 2017, 96, 094202. [CrossRef]

17. Buča, B.; Tindall, J.; Jaksch, D. Non-stationary coherent quantum many-body dynamics through dissipation. Nat. Commun. 2019, 10, 1730. [CrossRef]

18. Iemini, F.; Russomanno, A.; Keeling, J.; Schirò, M.; Dalmonte, M.; Fazio, R. Boundary time crystals. Phys. Rev. Lett. 2018, 121, 035301. [CrossRef]

19. Buča, B.; Jaksch, D. Dissipation induced nonstationarity in a quantum gas. Phys. Rev. Lett. 2019, 123, 260401. [CrossRef]

20. Basak, S.; Dahmen, K.; Carlson, E. Period multiplication cascade at the order-by-disorder transition in uniaxial random field XY magnets. Nat. Commun. 2020, 11, 4665. [CrossRef]

21. Giergiel, K.; Dauphin, A.; Lewenstein, M.; Zakrzewski, J.; Sacha, K. Topological time crystals. New J. Phys. 2019, $21,052003$. [CrossRef]

22. Chew, A.; Mross, D.F.; Alicea, J. Time-crystalline topological superconductors. Phys. Rev. Lett. 2020, 124, 096802. [CrossRef]

23. Keßler, H.; Cosme, J.G.; Hemmerling, M.; Mathey, L.; Hemmerich, A. Emergent limit cycles and time crystal dynamics in an atom-cavity system. Phys. Rev. A 2019, 99, 053605. [CrossRef]

24. Cosme, J.G.; Skulte, J.; Mathey, L. Time crystals in a shaken atom-cavity system. Phys. Rev. A 2019, 100, 053615. [CrossRef]

25. O'Sullivan, J.; Lunt, O.; Zollitsch, C.W.; Thewalt, M.L.W.; Morton, J.J.L.; Pal, A. Signatures of discrete time crystalline order in dissipative spin ensembles. New J. Phys. 2020, 22, 085001. [CrossRef]

26. Nurwantoro, P.; Bomantara, R.W.; Gong, J. Discrete time crystals in many-body quantum chaos. Phys. Rev. B 2019, 100, 214311. [CrossRef]

27. Sacha, K.; Zakrzewski, J. Time Crystals: A Review. Rep. Prog. Phys. 2017, 81, 016401. [CrossRef]

28. Else, D.V.; Monroe, C.; Nayak, C.; Yao, N.Y. Discrete Time Crystals. Annu. Rev. Condens. Matter Phys. 2020, 11, 467-499. [CrossRef]

29. Khemani, V.; Moessner, R.; Sondhi, S. A Brief History of Time Crystals. arXiv 2019, arXiv:1910.10745.

30. Sacha, K. Time Crystals; Springer Series on Atomic, Optical, and Plasma Physics; Springer: Cham, Switzerland, 2020; Volume 114, .

31. Watanabe, H.; Oshikawa, M.; Koma, T. Proof of the Absence of Long-range Temporal Orders in Gibbs States. J. Stat. Phys. 2020, 178, 926-935. [CrossRef]

32. Kozin, V.K.; Kyriienko, O. Quantum Time Crystals from Hamiltonians with Long-Range Interactions. Phys. Rev. Lett. 2019, 123, 210602. [CrossRef]

33. Öhberg, P.; Wright, E.M. Quantum Time Crystals and Interacting Gauge Theories in Atomic Bose-Einstein Condensates. Phys. Rev. Lett. 2019, 123, 250402. [CrossRef] [PubMed]

34. Khemani, V.; Moessner, R.; Sondhi, S. Comment on "Quantum Time Crystals from Hamiltonians with Long-Range Interactions". arXiv 2020, arXiv:2001.11037.

35. Syrwid, A.; Kosior, A.; Sacha, K. Comment on "Quantum Time Crystals and Interacting Gauge Theories in Atomic Bose-Einstein Condensates". Phys. Rev. Lett. 2020, 124, 178901. [CrossRef] [PubMed] 
36. Syrwid, A.; Kosior, A.; Sacha, K. Lack of a Genuine Time Crystal in a Chiral Soliton Model. Phys. Rev. Res. 2020, 2, 032038. [CrossRef]

37. Keßler, H.; Cosme, J.G.; Georges, C.; Mathey, L.; Hemmerich, A. From a continuous to a discrete time crystal in a dissipative atom-cavity system. New J. Phys. 2020, 22, 085002. [CrossRef]

38. Giergiel, K.; Tran, T.; Zaheer, A.; Singh, A.; Sidorov, A.; Sacha, K.; Hannaford, P. Creating big time crystals with ultracold atoms New J. Phys. 2020, 22, 085004. [CrossRef]

39. Yarloo, H.; Kopaei, A.E.; Langari, A. Homogeneous Floquet time crystal from weak ergodicity breaking. Phys. Rev. B 2020, 102, 224309. [CrossRef]

40. Huang, B.; Wu, Y.H.; Liu, W.V. Clean Floquet time crystals: Models and realizations in cold atoms. Phys. Rev. Lett. 2018, 120, 110603. [CrossRef]

41. Russomanno, A.; Iemini, F.; Dalmonte, M.; Fazio, R. Floquet time crystal in the Lipkin-Meshkov-Glick model. Phys. Rev. B 2017, 95, 214307. [CrossRef]

42. Zhang, J.; Hess, P.; Kyprianidis, A.; Becker, P.; Lee, A.; Smith, J.; Pagano, G.; Potirniche, I.D.; Potter, A.C.; Vishwanath, A.; et al. Observation of a discrete time crystal. Nature 2017, 543, 217-220. [CrossRef]

43. Choi, S.; Choi, J.; Landig, R.; Kucsko, G.; Zhou, H.; Isoya, J.; Jelezko, F.; Onoda, S.; Sumiya, H.; Khemani, V.; et al. Observation of discrete time-crystalline order in a disordered dipolar many-body system. Nature 2017, 543, 221-225. [CrossRef]

44. Gong, Z.; Hamazaki, R.; Ueda, M. Discrete time-crystalline order in cavity and circuit QED systems. Phys. Rev. Lett. 2018, 120, 040404. [CrossRef]

45. Lazarides, A.; Roy, S.; Piazza, F.; Moessner, R. Time crystallinity in dissipative Floquet systems. Phys. Rev. Res. 2020, 2, 022002. [CrossRef]

46. Gambetta, F.; Carollo, F.; Marcuzzi, M.; Garrahan, J.; Lesanovsky, I. Discrete time crystals in the absence of manifest symmetries or disorder in open quantum systems. Phys. Rev. Lett. 2019, 122, 015701. [CrossRef]

47. Yao, N.Y.; Nayak, C.; Balents, L.; Zaletel, M.P. Classical discrete time crystals. Nat. Phys. 2020, 16, 438-447. [CrossRef]

48. Mizuta, K.; Takasan, K.; Nakagawa, M.; Kawakami, N. Spatial-translation-induced discrete time crystals. Phys. Rev. Lett. 2018, 121, 093001. [CrossRef]

49. Pizzi, A.; Knolle, J.; Nunnenkamp, A. Period-n discrete time crystals and quasicrystals with ultracold bosons. Phys. Rev. Lett. 2019, 123, 150601. [CrossRef]

50. Pizzi, A.; Knolle, J.; Nunnenkamp, A. Higher-order and fractional discrete time crystals in clean long-range interacting systems. arXiv 2019, arXiv:1910.07539.

51. Zhu, B.; Marino, J.; Yao, N.Y.; Lukin, M.D.; Demler, E.A. Dicke time crystals in driven-dissipative quantum many-body systems. New J. Phys. 2019, 21, 073028. [CrossRef]

52. Surace, F.M.; Russomanno, A.; Dalmonte, M.; Silva, A.; Fazio, R.; Iemini, F. Floquet time crystals in clock models. Phys. Rev. B 2019, 99, 104303. [CrossRef]

53. Gambetta, F.; Carollo, F.; Lazarides, A.; Lesanovsky, I.; Garrahan, J.P. Classical stochastic discrete time crystals. Phys. Rev. E 2019, 100, 060105. [CrossRef]

54. Russomanno, A.; Notarnicola, S.; Surace, F.M.; Fazio, R.; Dalmonte, M.; Heyl, M. Homogeneous floquet time crystal protected by gauge invariance. Phys. Rev. Res. 2020, 2, 012003. [CrossRef]

55. Rovny, J.; Blum, R.L.; Barrett, S.E. Observation of discrete-time-crystal signatures in an ordered dipolar many-body system. Phys. Rev. Lett. 2018, 120, 180603. [CrossRef]

56. Pal, S.; Nishad, N.; Mahesh, T.; Sreejith, G. Temporal order in periodically driven spins in star-shaped clusters. Phys. Rev. Lett. 2018, 120, 180602. [CrossRef]

57. Šuntajs, J.; Bonča, J.; Prosen, T.; Vidmar, L. Quantum chaos challenges many-body localization. Phys. Rev. E 2020, 102, 062144. [CrossRef]

58. Kiefer-Emmanouilidis, M.; Unanyan, R.; Fleischhauer, M.; Sirker, J. Slow delocalization of particles in many-body localized phases. Phys. Rev. B 2021, 103, 024203. [CrossRef]

59. Kyprianidis, A.; Machado, F.; Morong, W.; Becker, P.; Collins, K.S.; Else, D.V.; Feng, L.; Hess, P.W.; Nayak, C.; Pagano, G.; et al. Observation of a prethermal discrete time crystal. arXiv 2021, arXiv:2102.01695.

60. Yu, W.C.; Tangpanitanon, J.; Glaetzle, A.W.; Jaksch, D.; Angelakis, D.G. Discrete time crystal in globally driven interacting quantum systems without disorder. Phys. Rev. A 2019, 99, 033618. [CrossRef]

61. Weinberg, M.; Ölschläger, C.; Sträter, C.; Prelle, S.; Eckardt, A.; Sengstock, K.; Simonet, J. Multiphoton interband excitations of quantum gases in driven optical lattices. Phys. Rev. A 2015, 92, 043621. [CrossRef]

62. Hu, J.; Feng, L.; Zhang, Z.; Chin, C. Quantum simulation of Unruh radiation. Nat. Phys. 2019, 15, 785-789. [CrossRef]

63. Chen, Y.Y.; Zhang, P.; Zheng, W.; Wu, Z.; Zhai, H. Many-body echo. Phys. Rev. A 2020, 102, 011301. [CrossRef]

64. Lyu, C.; Choudhury, S.; Lv, C.; Yan, Y.; Zhou, Q. Eternal discrete time crystal beating the Heisenberg limit. Phys. Rev. Res. 2020, 2, 033070. [CrossRef]

65. Barfknecht, R.E.; Rasmussen, S.E.; Foerster, A.; Zinner, N.T. Realizing time crystals in discrete quantum few-body systems. Phys. Rev. B 2019, 99, 144304. [CrossRef]

66. Barnes, E.; Nichol, J.M.; Economou, S.E. Stabilization and manipulation of multispin states in quantum-dot time crystals with Heisenberg interactions. Phys. Rev. B 2019, 99, 035311. [CrossRef] 
67. Fan, C.h.; Rossini, D.; Zhang, H.X.; Wu, J.H.; Artoni, M.; La Rocca, G. Discrete time crystal in a finite chain of Rydberg atoms without disorder. Phys. Rev. A 2020, 101, 013417. [CrossRef]

68. Li, B.; Van Dyke, J.S.; Warren, A.; Economou, S.E.; Barnes, E. Discrete time crystal in the gradient-field Heisenberg model. Phys. Rev. B 2020, 101, 115303. [CrossRef]

69. Dodonov, V.V. Current status of the dynamical Casimir effect. Phys. Scr. 2010, 82, 038105. [CrossRef]

70. Dodonov, V.V. Fifty years of the dynamical Casimir effect. Physics 2020, 2, 67-104. [CrossRef]

71. Dodonov, V.V.; Mendonça, J. Dynamical Casimir effect in ultra-cold matter with a time-dependent effective charge. Phys. Scr. 2014, T160, 014008. [CrossRef]

72. Mendonça, J.T.; Gammal, A. Time symmetry breaking in Bose-Einstein condensates. J. Phys. A Math. Theor. 2017, 50, 355501. [CrossRef]

73. Prosen, T. Exact time-correlation functions of quantum ising chain in a kicking transversal magnetic fieldspectral analysis of the adjoint propagator in heisenberg picture. Prog. Theor. Phys. Suppl. 2000, 139, 191-203. [CrossRef]

74. Prosen, T. General relation between quantum ergodicity and fidelity of quantum dynamics. Phys. Rev. E 2002, 65, 036208. [CrossRef]

75. Iadecola, T.; Hsieh, T.H. Floquet supersymmetry. Phys. Rev. Lett. 2018, 120, 210603. [CrossRef]

76. Guardado-Sanchez, E.; Brown, P.T.; Mitra, D.; Devakul, T.; Huse, D.A.; Schauß, P.; Bakr, W.S. Probing the quench dynamics of antiferromagnetic correlations in a 2D quantum Ising spin system. Phys. Rev. X 2018, 8, 021069. [CrossRef]

77. Labuhn, H.; Barredo, D.; Ravets, S.; De Léséleuc, S.; Macrì, T.; Lahaye, T.; Browaeys, A. Tunable two-dimensional arrays of single Rydberg atoms for realizing quantum Ising models. Nature 2016, 534, 667-670. [CrossRef]

78. Schauss, P. Quantum simulation of transverse Ising models with Rydberg atoms. Quantum Sci. Technol. 2018, 3, 023001. [CrossRef]

79. Simon, J.; Bakr, W.S.; Ma, R.; Tai, M.E.; Preiss, P.M.; Greiner, M. Quantum simulation of antiferromagnetic spin chains in an optical lattice. Nature 2011, 472, 307-312. [CrossRef]

80. Ippoliti, M.; Kechedzhi, K.; Moessner, R.; Sondhi, S.L.; Khemani, V. Many-body physics in the NISQ era: Quantum programming a discrete time crystal. arXiv 2020, arXiv:2007.11602.

81. Kyriienko, O.; Sørensen, A.S. Floquet quantum simulation with superconducting qubits. Phys. Rev. Appl. 2018, 9,064029. [CrossRef]

82. Lanyon, B.P.; Hempel, C.; Nigg, D.; Müller, M.; Gerritsma, R.; Zähringer, F.; Schindler, P.; Barreiro, J.T.; Rambach, M.; Kirchmair, G.; et al. Universal digital quantum simulation with trapped ions. Science 2011, 334, 57-61. [CrossRef]

83. Monroe, C.; Campbell, W.C.; Duan, L.M.; Gong, Z.X.; Gorshkov, A.; Hess, P.; Islam, R.; Kim, K.; Linke, N.; Pagano, G.; et al. Programmable quantum simulations of spin systems with trapped ions. arXiv 2019, arXiv:1912.07845.

84. Richerme, P. How to create a time crystal. Physics 2017, 10, 5. [CrossRef]

85. Gibney, E. The quest to crystallize time. Nature 2017, 543, 164-166. [CrossRef] [PubMed]

86. Estarellas, M.; Osada, T.; Bastidas, V.; Renoust, B.; Sanaka, K.; Munro, W.; Nemoto, K. Simulating complex quantum networks with time crystals. Sci. Adv. 2020, 6, eaay8892. [CrossRef]

87. Sun, G.; Eckardt, A. Optimal frequency window for Floquet engineering in optical lattices. Phys. Rev. Res. 2020, 2, 013241. [CrossRef]

88. Viebahn, K.; Minguzzi, J.; Sandholzer, K.; Walter, A.S.; Görg, F.; Esslinger, T. Suppressing dissipation in a Floquet-Hubbard system. Phys. Rev. X 2021, 11, 011057. 